\title{
Construction of a DNA library from chromosome 4 of rice (Oryza sativa) by microdissection
}

\author{
MAO Ying Wei, Si YuAN LIANG, Wen Qin SONG, \\ XiU LAN LI, Rui YANG CHEN* \\ Biology Department, Nankai University, Tianjin 300071, \\ China
}

\begin{abstract}
A simple method to create a chromosome-specific DNA library of rice, including microdissection, amplification, characterization and cloning, is described. Rice chromosome 4 from a metaphase cell has been isolated and amplified by the Linker Adapter PCR (LA-PCR). The PCR products were labeled as probes with DIG-11-dUTP using the random priming method. Southern blot analysis with rice genomic DNA and specific RFLP markers demonstrated that the PCR products were derived from rice chromosome 4. A large library comprising over 100,000 recombinant plasmid microclones from rice chromosome 4 was constructed. Colony hybridization showed that $58 \%$ of the clones contained single or low-copy sequences and $42 \%$ contained repetitive sequences. The size of inserts generated by PCR ranged from $140 \mathrm{bp}$ to $500 \mathrm{bp}$. This method will facilitate cloning of the specific chromosome DNA markers and important genes of rice.
\end{abstract}

Key words: Rice chromosome 4, DNA library, microdissection, $L A-P C R$.

\section{INTRODUCTION}

Rice is not only one of the most important food crops in the world, but also a model plant among the cereals for molecular genetics studies. It has 24 chromosomes and the smallest genome $\left(4 \times 10^{8} \mathrm{bp}\right)$ of any monocot. Some of important genes,

\footnotetext{
*Corresponding author, e-mail: chenry@public1.tpt.tj.cn
} 
for example, $\mathrm{Ph}$ (phenol staining), Gin-2 (Gall-midge resistance), Xa-1,2 (bacterial blight resistance) are located on chromosome 4 of rice[1]. Most important of all, sequencing of rice chromosome 4 will be carried out by the Rice Genome Project (RGP) in China.

With the development of the Human Genome Project (HGP) in recent years, analysis of restriction fragment length polymorphisms (RFLP) has become a powerful tool for gene tagging, gene manipulation and physical mapping; these methods are also widely used in rice genome research. McCouch et al. constructed the first RFLP map of rice in 1988[2]. Causse et al. enriched their molecular linkage map to 726 markers based on an interspecific backcross population. But there were only 84 markers mapped on chromosome 4[1]. Another linkage map of rice which was derived from a cross between Nipponbare (a japonica variety) and Kasalath (an indica variety) was constructed by Knrata et al. in Japan, and included a total of 1285 RFLP and random amplified polymorphism DNA (RAPD) markers. Using these probes they also constructed a physical map by YAC clones which has been arranged over $52 \%$ of the genome length. Their physical map of the rice chromosome 4 contained 114 DNA markers and 258 individual YACs which formed 17 contigs and covered $48 \%$ of the chromosome length (about $16.9 \mathrm{Mb}$ )[3, 4]. In China, Hong utilized bacterial artificial chromosomes (BACs) to build a large-scale contig map of rice including 631 contigs which covered $92 \%$ of the genome, based on fingerprinting and hybridization $[5,6]$.

However, most of these markers from RFLP maps in U.S.A. and Japan came from randomly selected genomic clones, cDNA clones and a few markers from other plants, such as oat, barley and maize. These random markers are distributed unevenly along the chromosomes. Many gaps in rice maps still need to be filled. In addition many of these clones are multi-copy probes, and few STS (Sequence-Tagged Site) markers are mapped. Because the genetic distance between two markers doesn't match the physical distance, we need more probes to saturate the map in order to reduce the errors. Cloning by microdissection and microcloning has proved to be an extremely useful technique to obtain more chromosome-specific molecular markers[7, 8]. Thus, our chromosome-specific library of rice can provide a large number of additional chromosomal assigned DNA markers which will facilitate map-based cloning and construction of a high resolution physical map of rice chromosome 4 .

\section{MATERIALS AND METHODS}

\section{Materials}

Orysa sativa L. guangluai NO.4 was kindly provided by Prof. Yang Jinshui (Fudan University). Eight RFLP markers, R2373, R1854, R288, C1100, C1016, C891, C335 and C445 were kindly provided by Prof. Sasaki, the head of Rice Genome Project (RGP) in Japan.

\section{Preparation of metaphase chromosome spreads and microdissection}

The procedures for preparation of metaphase cell and isolation of rice chromosome 4 were es- 


\section{Mao YW et al.}

sentially the same as those described by Song et al.[9].

\section{Pretreatment prior to LA-PCR}

Two rice chromosome 4 homologous from one cell were collected in a 0.5 -ml Eppendof tube by microdissection. $2 \mu$ l of Sau 3A I $1 \times$ multi-buffer (Promega) and $0.5 \mu \mathrm{l}$ of $10 \mathrm{mg} / \mathrm{ml}$ proteinase $\mathrm{K}$ solution were added. A clean tube without chromosome DNA was used as a negative control. Both were digested at $37{ }^{\circ} \mathrm{C}$ for $4 \mathrm{~h}$, then incubated at $78{ }^{\circ} \mathrm{C}$ for $30 \mathrm{~min}$ to inactivate proteinase K. Next $0.3 \mu$ l of $10 \times$ buffer B and $0.2 \mu$ l of 10 units/ $\mu$ l Sau 3A I (Promega) were added. The chromosomal DNA was digested at $37{ }^{\circ} \mathrm{C}$ for $4 \mathrm{~h}$, followed by incubation at $78{ }^{\circ} \mathrm{C}$ for $15 \mathrm{~min}$ to inactivate Sau 3A I.

The adapter was prepared by mixing two synthetic olignncleotides of which the 5'end was phosphorylated in an equimolar ratio: 24-mer sequence (1): 5'-CGGGAATTCTGGCTCTGCGACATG3', 10-mer sequence (2): 3'-CTGTACCTAG-5', then annealing at $58{ }^{\circ} \mathrm{C}$ for $1 \mathrm{~h}$. The adapter was as followed: 5'-CGGGAATTCTGGCTCTGCGACATG-3'

\section{$\uparrow$ EcoR I 3'-CTGTACCTAG-5'}

The DNA fragments cut by Sau 3A I were ligated with $0.6 \mu$ l of T4 DNA ligation $10 \times$ buffer, $1 \mu \mathrm{l}$ of 6 units $/ \mu$, T4 ligase (Promega) at $12-14{ }^{\circ} \mathrm{C}$ for $14-16 \mathrm{~h}$.

\section{LA-PCR}

After ligation, PCR reaction mixture $(100 \mu \mathrm{l})$ was added $[1 \times$ PCR reaction buffer, $2 \mathrm{mM}$ $\mathrm{MgCl}_{2}, 0.2 \mathrm{mM}$ each dNTP, 5 units Taq polymerase (Goldworld), $0.2 \mu \mathrm{M}$ primer]. The 24-mer sequence(1) was used as the primer. The following PCR conditions were used: first at $72{ }^{\circ} \mathrm{C}$ for $10 \mathrm{~min}$ to fill the recessed $3^{\prime}$ end of the adapter, then 35 cycles at $96{ }^{\circ} \mathrm{C}$ for $1 \mathrm{~min}, 54^{\circ} \mathrm{C}$ for $1 \mathrm{~min}, 72{ }^{\circ} \mathrm{C}$ for $1.5 \mathrm{~min}$, finally an extension at $72{ }^{\circ} \mathrm{C}$ for $5 \mathrm{~min} .1 \mu \mathrm{l}$ of primary product was amplified in a secondary PCR reaction identical to that described above without the filling reaction.

\section{Probe preparation}

Rice genomic DNA was extracted by the CTAB method[10]. The purified PCR products and rice genomic DNA were labeled by the random priming method, according to the Direction of DIG DNA Labeling and Detection Kit (Boehring Mannhein).

\section{Southern blot hybridization}

First, labeled total rice DNA was hybridized to a filter containing the secondary PCR products and rice genomic DNA. Second, the purified secondary PCR products were labeled as probes to hybridize with eight RFLP markers of rice chromosome 4: R2373, R1854, C1100, C1016, C891, C445, C335, R288. The method was described in Molecular Cloning[11].

\section{Cloning into pUC19}

After PCR, the amplified products were purified by phenol/chloroform extraction, digested with EcoR I, and ligated to dephosphorylated pUC19 vector with T4 ligase. One-fifth of the ligation mixture was used in transformation of the highly efficient E.coli DH5 a competent cells. One-fifth of the transformed mixture was plated onto LB medium with ampicillin and IPTG-X-gal.

\section{Characterization of microclones}

After transformation, white colonies were isolated for initial characterization. Rice total DNA was labeled with $a{ }^{32} \mathrm{P}-\mathrm{dCTP}$ and hybridized to filters containing recombinant microclones, as described by Kao et al.[12]. The single copy probe C335 and rice genomic DNA as controls. The size of inserts contained in the microclones was estimated by PCR in which specific primers flanking the cloning sites of pUC19 were used to amplify the insert sequence in each clone. Two 20-mers were used 
DNA library from chromosome 4 of rice

as the primers: 5'-ACAGGAAACAGCTATGACCA-3' and 5'-CGTTGTAAAACGACGGCCAG3 '[13].

\section{RESULTS}

\section{Microdissection of rice chromosome 4}

Rice chromosome 4 was isolated from one metaphase cell (Fig 1) using a micromanipulator combined with a Nikon microscope and a $1 \mu \mathrm{m}$ tip-glass needle. Rice chromosome 4 is easy to identify, as it is submetacentric and the 4th longest chromosome.

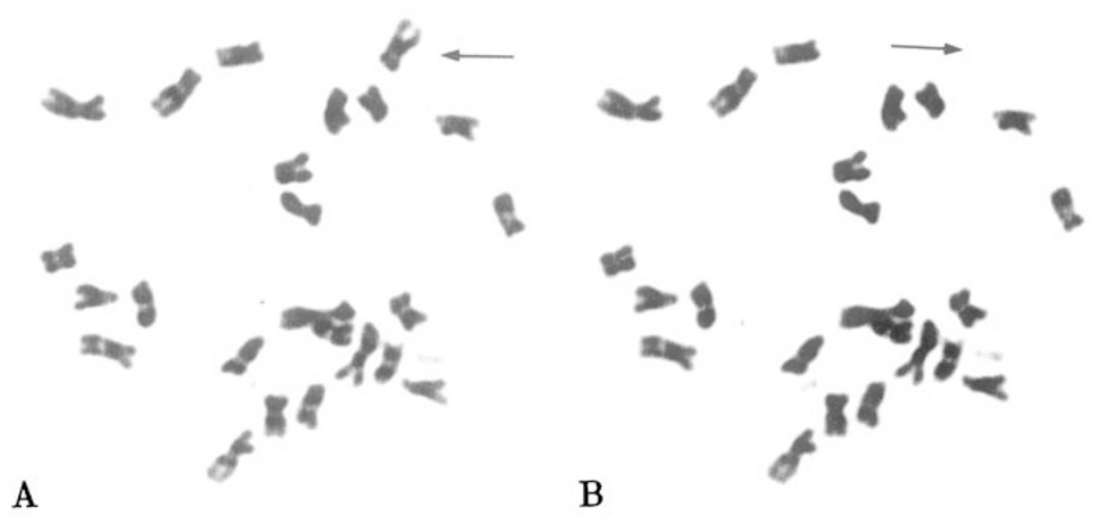

Fig 1. Microdissection of rice chromosome 4.

A. Before microdissection of rice chromosome, arrow indicates chromosome 4 .

B. After microdissection of rice chromosome 4 .

\section{Amplification of microdissected chromosome 4 using Sau 3A I tinker adapter}

From the 1.2\% agarose gel electrophoresis pattern (Fig 2), the primary and secondary PCR products were smears of fragments ranging from $250 \mathrm{bp}-2000 \mathrm{bp}$, and there was no detectable amplification of extraneous DNA in negative control. Appearance of specific bands was not observed, which suggests no preferential amplification of a subset of DNA sequences. When stained with ethidium bromide, the amount of amplified DNA in the secondary reaction is estimated in the $\mu \mathrm{g}$ range, which ensures enough material for probes.

\section{Characterization of PCR products by Southern blot hybridization}

Southern blot analysis showed strong hybridization signals between rice genomic probe DNA and PCR products in lane 3, whereas no signal is seen in the negative control in lane 4; this confirmed that rice DNA sequences have been amplified (Fig 3). Additionally, pooled PCR products were used as probes to hybridize with eight 
RFLP markers of rice chromosome 4: R2373, R1854, C1100, C1016, C891, C445, C335, R288. As shown in two recent works[3, 4], these eight DNA markers were selected specially for their even distribution along the linkage map of rice chromosome 4. Among these markers C445 and R2373 were located at the two ends of the linkage map and R2373, R1854, C1100, C1016, C891, R288 were used to construct a rice physical map[3]. Furthermore, C1100, C1016, C891, C445, C335 were selected from a rice cDNA library, and parts of their sequences were released on Gene Bank. So these eight probes are site-specific unique sequences. All of these markers except for R288 demonstrated intensive hybridization signals with amplified products (Fig 4). These results gave further evidence that these PCR products were derived from rice chromosome 4 .

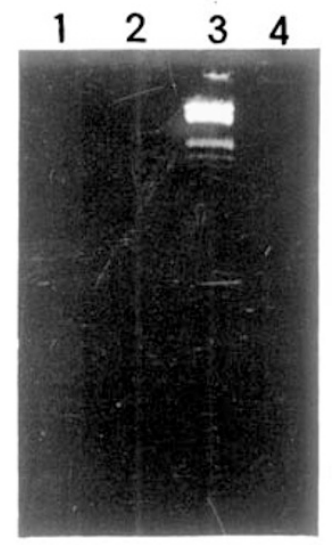

Fig 2. Amplified products of LA-PCR

1: Primary PCR products

2: Secondary PCR products

3: $\lambda$ /Hind III + EcoR I

4: Negative control

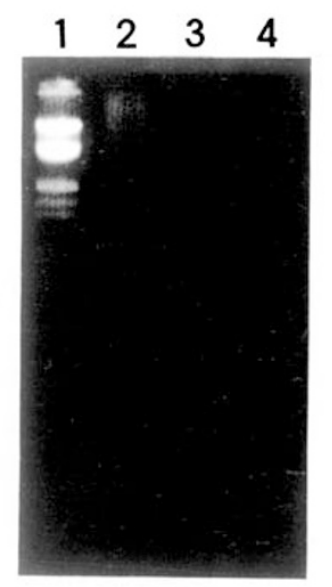

A

\section{$\begin{array}{lll}2 & 3 & 4\end{array}$}

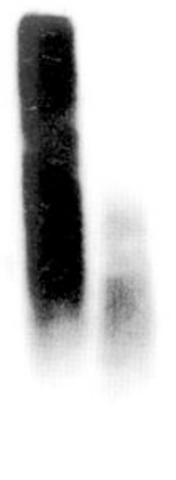

B

Fig 3. Southern blot hybridization analysis between rice total DNA and PCR products
A. Before hybridization
B. After hybridization
1: $\lambda /$ Hind III + EcoR I
2: Rice genomic DNA
3: Secondary PCR products
4: Negative control

\section{Characterization of microclones}

Analysis of 500 clones with colony hybridization revealed that about $42 \%$ of them 
DNA library from chromosome 4 of rice

hybridized either strongly (highly repetitive sequences) or less strongly (moderately repetitive or slightly repetitive sequences) and 58\% showed no detectable hybridization (containing unique or very low-copy sequences) (data was not shown). We selected 66 microclones randomly from the recombinant clones to determine their insert size, which ranged from 140 to $500 \mathrm{bp}$ with a mean length of $300 \mathrm{bp}$ (Fig 5).

Fig 4. Southern hybridization analysis between PCR products and RFLP markers

A. Before hybridization

B. After hybridization

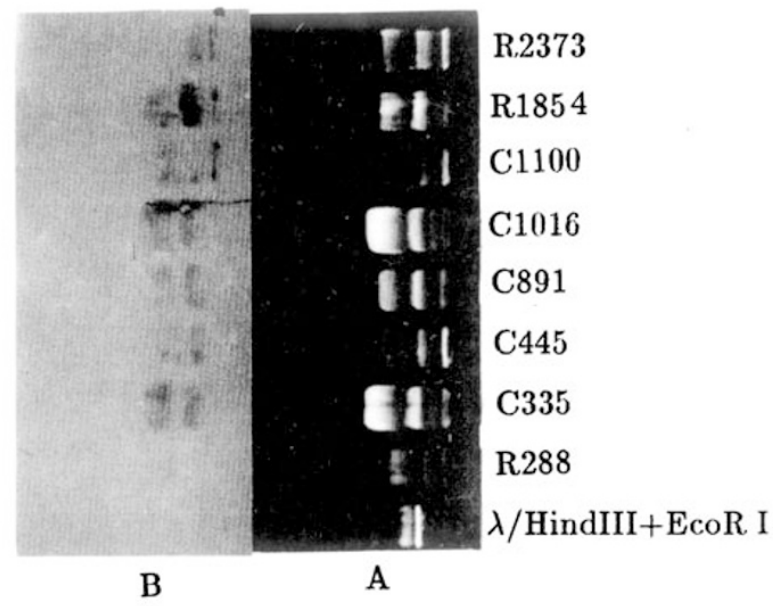

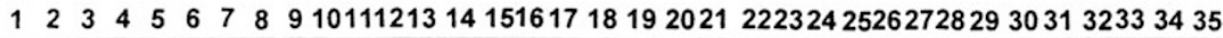

A

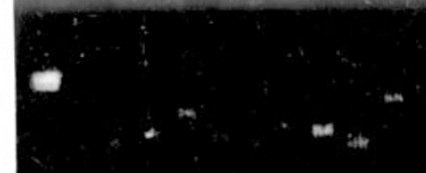

B

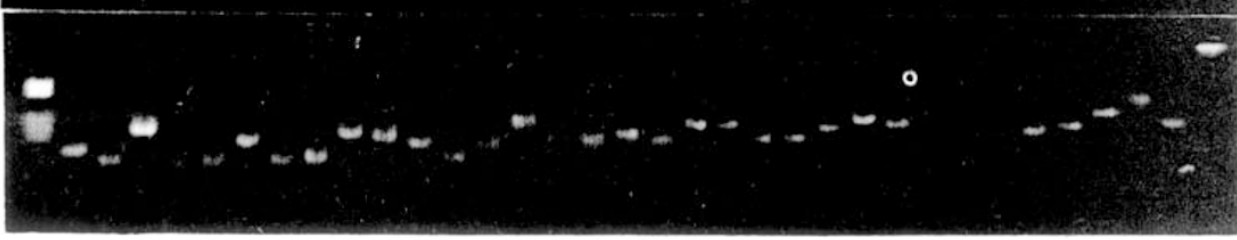

Fig 5. Inserts of recombined plasmids

A and B represent different sets of clones

1: pBR322/Hae III; 2-34: Inserts of microclones; $35: \lambda /$ HindIII + EcoR I 
Mao YW et al.

\section{DISCUSSION}

Because of the lack of special reagents such as human-mouse hybrid cell lines, it is difficult to verify chromosome specificity of PCR products in plant. Southern blot hybridization with total genomic DNA can only prove their origin from certain plants but not accurately from specific chromosomes[14]. Furthermore, some researchers using FISH found that the hybridization signals were dispersed on each chromosome due to repetitive sequences in higher plants[15, 16]. In contrast it is quick and simple to identify where the PCR products come from using specific DNA markers, which have been mapped on designated chromosomes. Cheng et al. succeeded in proving that their PCR products were derived from rice chromosome 5 using STS and microsatellite markers[17]. In order to verify the chromosome-specific origin of our library, firstly Southern blot analysis with genomic DNA was used to prove our products really contained rice sequences. Then, we carried out hybridization with eight RFLP markers that were site-specific single copy probes. The results indicated that our library not only originated from rice chromosome 4 but also covered a fairly high proportion of it. The fluorescence in situ hybridization (FISH) using the secondary PCR products of rice chromosome 4 as probe was also carried out and the same result was gotten as Hernould et al.[15] that hybridization signals were distributed on every chromosome of rice including chromosome 4 (data not shown). Maybe the dispersion of in situ hybridization signals was a characterization of higher plants because of high proportion of repetitive sequences. However, this is still a problem deserving of further discussion. Individual and multiple chromosome specific unique probe fluorescent images of high quality by FISH are certainly good means to double check the target chromosome, which we would like to perform in the future.

It is relatively simple and efficient to isolate a large number of microclones containing unique sequences by colony hybridization. $58 \%$ of the clones in our library can be expected to contain single or low-copy sequences which is similar to the content of such sequences in the rice genome (50\%). This result demonstrates that LA-PCR had no preferences for repetitive sequences. Thus, thousands of microclones with unique sequences can be conveniently isolated and used in screening other rice genomic libraries for the corresponding genes.

Even though RFLP linkage maps of rice have been completed in Japan and America, and the construction of a rice physical map is being carried out, there are still many gaps in the maps. A saturation map of every small genomic region is indispensable for map-based cloning of genes of interest and filling up gaps in a physical map. Currently there are 163,215 ESTs (Expressed Sequence Tags) and about 30,000 STS markers located on human chromosomes[18]. In the rice genome, another 900 RFLP markers were added to the linkage map after the Japanese RGP published their physical map[3]. However, compared with the progress of the Human Genome Project, DNA markers to the rice linkage map have not progressed so far. Thus, it is necessary to construct DNA libraries of each chromosome and to isolate 
large numbers of unique sequence probes from defined chromosomes. The probes from our library can be used to directly create "Sequence-Tagged Sites" (STS) for rice chromosome 4 . In addition, the probes can be conveniently used to screen YAC libraries with large inserts. If rice chromosome 4 contains $35.4 \mathrm{Mb}$, it appears feasible to use unique-sequence microclones to isolate 70-100 corresponding YACs with average insert size of $500 \mathrm{~kb}$, to construct contigs for entire chromosome. Our library can provide enough genomic landmarks for high-resolution physical map of the entire rice chromosome 4 . The probes can also be used to screen various cDNA libraries for isolating expressed sequences from rice chromosome 4. Most of cDNA sequences have functional significance and can be regarded as candidate genes. Finally, our results will provide a great deal of important molecular information, such as telomere and centromere sequences. These sequences will facilitate locating these sites on physical map and research on the function of plant chromosome.

\section{ACKNOWLEDGEMENTS}

We gratefully acknowledge Prof. Sasaki, the head of Rice Genome Project (RGP) of Japan for providing specific RFLP markers of rice chromosome 4, and Dr. Peter McInerney for assistance in revising the English language version of the manuscript. This project was supported by grants from National Committee of Science and Technology and Tianjin Natural Scientific Foundation.

\section{REFERENCES}

[1] Causse MA, Fulton TM, Cho YG, et al. Saturated molecular map of the rice genome based on an interspecific backcross population. Genetics 1994; 138:1251-74.

[2] McCouch SR, Kochert G, Yu ZH, et al. Molecular mapping of rice chromosomes. Theor Appl Genet 1988; 76:815-29.

[3] Kurata N, Umehara Y, Tanoue H, et al. Physical mapping of the rice genome with YAC clones. Plant Molecular Biology 1997; 35:101-13.

[4] Koike K, Yoshino K, Sue N, et al. Physical mapping of rice chromosome 4 and 7 using YAC clones. DNA Research 1997; 4(1):27-33.

[5] Hong GF. A rapid and accurate strategy for rice contig map construction by combination of fingerprinting and hybridization. Plant Molecular Biology 1997; 35:129-33.

[6] Hong GF. Contigs of rice genome. Life Science 1998; 10:1-3.

[7] Fiedler W, Claussen U, Ludecke HJ, et al. New markers for the cneurofibromatosis-2 region generated by microdissection of chromosome 22 . Genomics $1991 ; \mathbf{1 0}: 786-91$.

[8] Jung C, Claussen U, Horsthemke B, et al. A DNA library from an individual Beta patellaris chromosome conferring nematode resistance obtained by microdissection of meiotic metaphase chromosomes. Plant Molecular Biology 1992; 20:503-11.

[9] Song WQ, Chui XQ Xu WS, et al. Microdissection and polymerase chain reaction (PCR) amplification from distal end of the long arm of the M-chromosome in Vicia faba. Chinese Sci Bull 1996; 41(4):361-3.

[10] Dellaporta, S.L, Wood, et al. A plant DNA minipreparation Versiona II. Plant Mol.Biol Rep 1983; 1(14):19.

[11] Sambrook J, Fritsch EF, Maniatis T. Molecular Cloning. Cold Spring Harbor Laboratory Press 1989. 
[12] Kao FT, Hartz JA, Martha, et al. Isolation and chromosomal location of unique DNA sequences from a human genomic library. Proc Natl Acad Sci USA 1982; 79:865-9.

[13] Kao FT and Yu TW, Chromosome microdissection and cloning in human genome and genetic disease analysis. Proc Natl Acad Sci USA 1991: 88:1844-8.

[14] Zhou YH, Dang BY, Hu ZM, et al. Microdissection and PCR ampification of single soybean chromosome. Acta Botanica Sinica 1998; 40(2):144-50.

[15] Hernould M, Glimelius K, Venskens J, et al. Microdissection and amplification of coding sequences from a chromosome fragment restoring male fertility in alloplasmic male-sterile. The Plant Journal 1997; 12(3):703-9.

[16] Vega JM, Abbo S, Feldman M, et al. Chromosome painting in plants: In situ hybridization with a DNA probe from a specific microdissected chromosome arm of common wheat. Proc Natl Acad Sci USA 1994; 91:12041-5.

[17] Cheng ZK, Yan HH, Dang BY, et al. Applications of isotetrasome from the short arm of rice chromosome 5 in microdissection. Chinese Sci Bull 1998; 43:272-6.

[18] Schuler GD, Boguski MS, Stewart EA, et al. A gene map of the human genome. Science 1996; 274:540-6.

\section{Received May-29-1998. Revised Aug-21-1998. Accepted Sept-16-1998.}

